\title{
Probing non perturbative QCD with the cusp effect in kaon decays by NA48
}

\author{
Dmitry Madigozhin *i \\ JINR \\ E-mail: madigo@sunse.jinr.ru
}

We report the results from a study of the full sample of $\sim 6.031 \times 10^{7} K^{ \pm} \rightarrow \pi^{ \pm} \pi^{0} \pi^{0}$ decays recorded by the NA48/2 experiment at the CERN SPS. The cusp-like anomaly of the $\pi^{0} \pi^{0}$ invariant mass $\left(M_{00}\right)$ distribution has been interpreted as an effect of the final state pions scattering in $K^{ \pm} \rightarrow \pi^{ \pm} \pi^{+} \pi^{-}$decay. Fits to the $M_{00}$ distribution using two different theoretical formulations provide the presently most precise determination of $a_{0}-a_{2}$, the difference between the $\pi \pi$ S-wave scattering lengths in the isospin $I=0$ and $I=2$ states. Less precise determination of $a_{2}$ also has been done.

2009 KAON International Conference

June 09 - 12, 2009

Tsukuba, Japan

* Speaker.

$\dagger$ on behalf of NA48/2 collaboration 


\section{Introduction}

The main purpose of the NA48/2 experiment at the CERN SPS was to search for direct CP violation in $K^{ \pm}$decay to three pions [1, 2, 3]. Data were collected in 2003-2004, providing large samples of fully reconstructed $K^{ \pm} \rightarrow \pi^{ \pm} \pi^{+} \pi^{-}$and $K^{ \pm} \rightarrow \pi^{ \pm} \pi^{0} \pi^{0}$ decays.

From the analysis of the data collected in 2003, we have already reported the observation of a cusp-like anomaly in the $\pi^{0} \pi^{0}$ invariant mass $\left(M_{00}\right)$ distribution of $K^{ \pm} \rightarrow \pi^{ \pm} \pi^{0} \pi^{0}$ decays in the region around $M_{00}=2 m_{+}$, where $m_{+}$is the charged pion mass [4]. The existence of this threshold anomaly had been first predicted in 1961 by Budini and Fonda [5]. More recently, Cabibbo [6] has proposed an interpretation of the cusp-like anomaly, expressing the $K^{ \pm} \rightarrow \pi^{ \pm} \pi^{0} \pi^{0}$ decay amplitude in terms of the $\pi^{+} \pi^{-} \rightarrow \pi^{0} \pi^{0}$ amplitude at threshold, $a_{x}$. In the limit of exact isospin symmetry $a_{x}$ can be written as $\left(a_{0}-a_{2}\right) / 3$, where $a_{0}$ and $a_{2}$ are the S-wave $\pi \pi$ scattering lengths in the isospin $I=0$ and $I=2$ states, respectively.

Here we report the results from a study of the final sample of $\sim 6.031 \times 10^{7} K^{ \pm} \rightarrow \pi^{ \pm} \pi^{0} \pi^{0}$ decays. Best fits to two independent theoretical formulations of rescattering effects in $K^{ \pm} \rightarrow \pi^{ \pm} \pi^{+} \pi^{-}$ and $K^{ \pm} \rightarrow \pi^{ \pm} \pi^{+} \pi^{-}$decays ([7] and [8,9]) provide a precise determination of $a_{0}-a_{2}$, and an independent, though less precise, determination of $a_{2}$.

A detailed description of the beams and detector elements is available in $[1,2,3,10]$. The two simultaneous beams are produced by $400 \mathrm{GeV} / \mathrm{c}$ protons impinging on a $40 \mathrm{~cm}$ long Be target. Particles of opposite charge with a central momentum of $60 \mathrm{GeV} / c$ and a momentum band of $\pm 3.8 \%$ ( $r m s$ ) produced at zero angle are selected by two systems of dipole magnets forming "achromats" with null total deflection, focusing quadrupoles, muon sweepers and collimators. The decay volume is a $114 \mathrm{~m}$ long vacuum tank.

Charged particles from $K^{ \pm}$decays are measured by a magnetic spectrometer consisting of four drift chambers and a large-aperture dipole magnet [10]. A $16 \mathrm{~cm}$ diameter aluminium vacuum tube centred on the beam axis runs through central holes in the detector components. Charged particles are magnetically deflected in the horizontal plane by an angle corresponding to a transverse momentum kick of $120 \mathrm{MeV} / c$. The momentum resolution of the spectrometer is $\sigma(p) / p=1.02 \% \oplus 0.044 \% p(p$ in $\mathrm{GeV} / c)$. The magnetic spectrometer is followed by a scintillator hodoscope.

A liquid Krypton calorimeter ( $\mathrm{LKr}$ ) [11] is used to reconstruct $\pi^{0} \rightarrow \gamma \gamma$ decays. It is an almost homogeneous ionization chamber with an active volume of $\sim 10 \mathrm{~m}^{3}$ of liquid krypton, segmented transversally into $2 \mathrm{~cm} \times 2 \mathrm{~cm}$ projective cells. The calorimeter is $27 X_{0}$ thick and has an energy resolution $\sigma(E) / E=0.032 / \sqrt{E} \oplus 0.09 / E \oplus 0.0042$ ( $\mathrm{E}$ in $\mathrm{GeV}$ ). The space resolution for single electromagnetic showers can be parameterized as $\sigma_{x}=\sigma_{y}=0.42 / \sqrt{E} \oplus 0.06 \mathrm{~cm}$ for each transverse coordinate $x, y$. An additional hodoscope consisting of a plane of scintillating fibers is installed in the $\mathrm{LKr}$ calorimeter at a depth of $\sim 9.5 X_{0}$.

\section{Event selection and reconstruction}

Events with at least one charged particle track having a momentum above $5 \mathrm{GeV} / c$, and at least four energy clusters in the LKr, each consistent, in terms of size, time and energy, with the electromagnetic shower produced by a photon of energy above $3 \mathrm{GeV}$, are selected for further 
analysis. The distance between any two photons in the LKr is required to be larger than $10 \mathrm{~cm}$, and the distance between each photon and the impact point of any track on the LKr front face must exceed $15 \mathrm{~cm}$. Fiducial cuts on the distance of each photon from the LKr edges and centre are also applied in order to ensure full containment of the electromagnetic showers. The minimum distance between the photon and the nearest LKr dead cell affected by readout problems ("dead cell") is required to be at least $2 \mathrm{~cm}$.

We assume that each possible pair of photons originates from a $\pi^{0} \rightarrow \gamma \gamma$ decay and we calculate the distance $D_{i j}$ between the $\pi^{0}$ decay vertex and the LKr front face: $D_{i j}=\frac{\sqrt{E_{i} E_{j}} R_{i j}}{m_{0}}$, where $E_{i}, E_{j}$ are the energies of the $i$-th and $j$-th photon, respectively, $R_{i j}$ is the distance between their impact points on $\mathrm{LKr}$, and $m_{0}$ is the $\pi^{0}$ mass. Among all possible $\pi^{0}$ pairs, only those with $D_{i j}$ values differing by less than $500 \mathrm{~cm}$ are retained further, and the distance $D$ of the $K^{ \pm}$decay vertex from the $\mathrm{LKr}$ is taken as the arithmetic average of the two $D_{i j}$ values. The reconstructed distance of the decay vertex from the LKr is further required to be at least $2 \mathrm{~m}$ downstream of the final beam collimator to exclude $\pi^{0}$-mesons produced from beam particles interacting in the collimator material.

Because of the long decay volume, a photon emitted at small angle to the beam axis may cross the aluminium vacuum tube in the spectrometer or the DCH1 central flange, and convert to $e^{+} e^{-}$ before reaching the LKr. To reject such a photon, for each LKr cluster we require that its distance from the nominal beam axis at the DCH1 plane must be $>11 \mathrm{~cm}$, assuming an origin on axis at $D-400 \mathrm{~cm}$.

Each surviving $\pi^{0}$ pair is then combined with a charged particle track, assumed to be a $\pi^{ \pm}$. Only those combinations with a total $\pi^{ \pm} \pi^{0} \pi^{0}$ energy between 54 and $66 \mathrm{GeV}$ are retained. For each combination, the energy-weighed average coordinates (center-of-gravity, COG) are calculated at each DCH plane using the photon impact points on $\mathrm{LKr}$ and the track parameters measured before the magnet. Acceptance cuts are then applied on the COG radial position on each DCH plane. In addition, we require a minimal separation between the COG and the charged track coordinates, as measured in each DCH plane. Limits for these cuts depend on the COG and track impact point distributions at each drift chamber.

For events with more than one accepted track-cluster combination, the $K^{ \pm} \rightarrow \pi^{ \pm} \pi^{0} \pi^{0}$ decay is selected as the $\pi^{ \pm} \pi^{0} \pi^{0}$ combination minimizing a quality estimator based on the difference $\Delta D$ of the two $D_{i j}$ values and the difference $\Delta M$ between the $\pi^{ \pm} \pi^{0} \pi^{0}$ invariant mass and the nominal $K^{ \pm}$ mass $\left(\frac{\Delta D}{r m s_{D}(D)}\right)^{2}+\left(\frac{\Delta M}{r m s_{M}(D)}\right)^{2}$, where the space and mass resolutions $r m s_{D}, r m s_{M}$ are functions of $D$, as obtained from the measured $\Delta D$ and $\Delta M$ distributions.

Fig. 1 shows the distribution of the square of the $\pi^{0} \pi^{0}$ invariant mass, $M_{00}^{2}$, for the final event sample. This distribution is displayed with a bin width of $0.00015\left(\mathrm{GeV} / \mathrm{c}^{2}\right)^{2}$. The cusp at $M_{00}^{2}=\left(2 m_{+}\right)^{2}=0.07792\left(\mathrm{GeV} / c^{2}\right)^{2}$ is clearly visible.

\section{Determination of the $\pi \pi$ scattering lengths $a_{0}$ and $a_{2}$}

The $\pi \pi$ scattering lengths $a_{0}$ and $a_{2}$ have been determined by fits of the full data set to two theoretical approaches: the Cabibbo-Isidori (CI) formulation [7], and the more recent Bern-Bonn (BB) formulation [8]. The BB approach uses a non-relativistic Lagrangian framework, which au- 


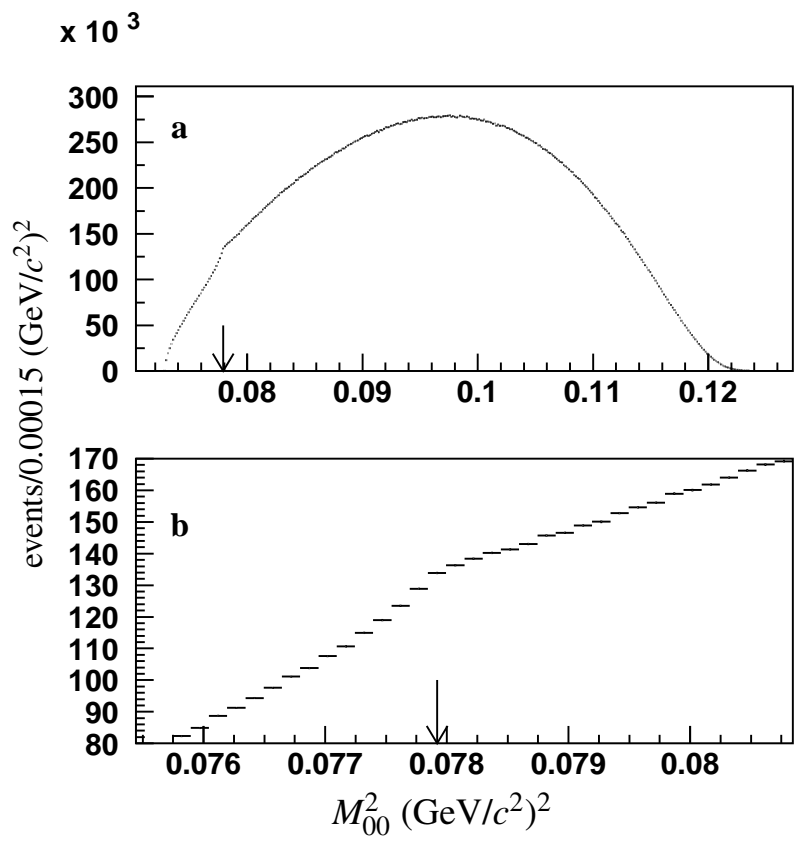

Figure 1: a: distribution of $M_{00}^{2}$, the square of the $\pi^{0} \pi^{0}$ invariant mass; $\mathbf{b}$ : enlargement of a narrow region centred at $M_{00}^{2}=\left(2 m_{+}\right)^{2}$ (this point is indicated by the arrow). The statistical error bars are also shown in these plots.

tomatically satisfies unitarity and analiticity constraints, and allows one to include electromagnetic contributions in a standard way [9]. In the CI approach, the structure of the cusp singularity is treated using unitarity, analiticity and cluster decomposition properties of the $S$-matrix. The decay amplitude is expanded in powers of $\pi \pi$ scattering lengths up to order (scattering length) ${ }^{2}$, and electromagnetic effects are omitted. To take them into account, we use the BB electromagnetic effect to correct $\mathrm{CI}$ amplitude.

In all fits we also need information on the $K^{ \pm} \rightarrow \pi^{ \pm} \pi^{+} \pi^{-}$decay amplitude. To this purpose, we use a sample of $4.709 \times 10^{8} K^{ \pm} \rightarrow \pi^{ \pm} \pi^{+} \pi^{-}$decays which are also measured in this experiment [13].

In the Cabibbo-Isidori (CI) formulation [7] the weak amplitudes for $K^{ \pm} \rightarrow \pi^{ \pm} \pi^{0} \pi^{0}$ and $K^{ \pm} \rightarrow$ $\pi^{ \pm} \pi^{+} \pi^{-}$decay at tree level (without pion scattering) are written as

$$
\begin{gathered}
\mathscr{M}_{0}=1+\frac{1}{2} g_{0} u+\frac{1}{2} h_{0} u^{2}+\frac{1}{2} k_{0} v^{2}, \\
\mathscr{M}_{+}=A_{+}\left(1+\frac{1}{2} g u+\frac{1}{2} h u^{2}+\frac{1}{2} k v^{2}\right),
\end{gathered}
$$

respectively. In Eq. $3.1 u=\left(s_{3}-s_{0}\right) / m_{+}^{2}$, where $s_{0}=\left(m_{K}^{2}+2 m_{0}^{2}+m_{+}^{2}\right) / 3$, while in Eq. $3.2 u=$ $\left(s_{3}-s_{+}\right) / m_{+}$, where $s_{+}=m_{K}^{2} / 3+m_{+}^{2}$; for both amplitudes $s_{i}=\left(P_{K}-P_{i}\right)^{2}$, where $P_{K}\left(P_{i}\right)$ is the $K(\pi)$ four-momentum and $i=3$ corresponds to the odd pion $\left(\pi^{ \pm}\right.$from $K^{ \pm} \rightarrow \pi^{ \pm} \pi^{0} \pi^{0}, \pi^{\mp}$ from $K^{ \pm} \rightarrow \pi^{ \pm} \pi^{+} \pi^{-}$decay), and $v=\left(s_{1}-s_{2}\right) / m_{+}^{2}$. 
In the CI case, $\mathscr{M}_{+}$parameters enter into the $K^{ \pm} \rightarrow \pi^{ \pm} \pi^{0} \pi^{0}$ amplitude only via the complete $\mathscr{M}_{+}$expression. So we use parametrisation (3.2) with the parameter values, fixed from a separate fit to the $K^{ \pm} \rightarrow \pi^{ \pm} \pi^{+} \pi^{-}$decay Dalitz plot [13].

In the fits to the $M_{00}^{2}$ distribution from $K^{ \pm} \rightarrow \pi^{ \pm} \pi^{0} \pi^{0}$ decay, the free parameters are $\left(a_{0}-\right.$ $\left.a_{2}\right) m_{+}, a_{2} m_{+}, g_{0}, h_{0}$, and an overall normalization constant. The coefficient $k_{0}$ value is determined independently from the Dalitz plot distribution of $K^{ \pm} \rightarrow \pi^{ \pm} \pi^{0} \pi^{0}$ decays. For CI formulation, the value $k_{0}=0.0099$ is kept fixed in the fits.

All fits are performed over the $M_{00}^{2}$ interval from 0.074094 to $0.104244\left(\mathrm{GeV} / c^{2}\right)^{2}$. For any given value of the generated $\pi^{0} \pi^{0}$ invariant mass the Monte-Carlo simulation provides the detection probability and the distribution function for the reconstructed value of $M_{00}^{2}$. This allows the transformation of any theoretical distribution into an expected distribution which can be compared directly with the measured one. Pionium formation and its dominating decay to $\pi^{0} \pi^{0}$ together with the resonant electromagnetic effects near the pionium production threshold are taken into account in the fit by multiplying the expected content (without electromagnetic correction) of the bin centred at $M_{00}^{2}=4 m_{+}^{2}$ by $\left(1+f_{\text {atom }}\right)$, where $f_{\text {atom }}$ is a free parameter.

In order to obtain a precision of the fit results which matches the BB approximation level, for the fits with BB formulation the value of each coefficient of the $K^{ \pm} \rightarrow \pi^{ \pm} \pi^{+} \pi^{-}$tree-level amplitude is obtained together with the parameters $\left(a_{0}-a_{2}\right) m_{+}, a_{2} m_{+}, g_{0}, h_{0}$ from the simultaneous fits to two distributions: the $M_{00}^{2}$ distribution from $K^{ \pm} \rightarrow \pi^{ \pm} \pi^{0} \pi^{0}$ and the $M_{ \pm \pm}^{2}$ distribution from $K^{ \pm} \rightarrow \pi^{ \pm} \pi^{+} \pi^{-}$decay, obtained as a projection of the Dalitz plot described in ref. [13]. The fixed value $k_{0}=0.0085$, determined independently from a separate fit to the Dalitz plot distribution of $K^{ \pm} \rightarrow \pi^{ \pm} \pi^{0} \pi^{0}$ decays, is used in the fits.

We have also performed fits using the constraint between $a_{2}$ and $a_{0}$ predicted by analyticity and chiral symmetry [15] (we refer to this constraint as the ChPT constraint):

$$
a_{2} m_{+}=(-0.0444 \pm 0.0008)+0.236\left(a_{0} m_{+}-0.22\right)-0.61\left(a_{0} m_{+}-0.22\right)^{2}-9.9\left(a_{0} m_{+}-0.22\right)^{3}
$$

The contributions to systematic uncertainty due to the trigger inefficiency, simulation quality of acceptance, $\mathrm{LKr}$ resolution and nonlinearity, hadronic showers and beam characteristics have been investigated. The most important source of external error is the value of $\left|A_{+}\right|$(see Eq. (3.2)), obtained from the measured ratio of the $K^{ \pm} \rightarrow \pi^{ \pm} \pi^{+} \pi^{-}$and $K^{ \pm} \rightarrow \pi^{ \pm} \pi^{0} \pi^{0}$ decay rates, $R=$ $3.175 \pm 0.050[12]$.

\section{Results}

The BB formulation with radiative corrections [9] provides presently the most complete description of rescattering effects in $K \rightarrow 3 \pi$ decay. For this reason we use the results from the fits to this formulation to present our final results on the $\pi \pi$ scattering lengths:

$$
\begin{array}{r}
\left(a_{0}-a_{2}\right) m_{+}=0.2571 \pm 0.0048(\text { stat. }) \pm 0.0025(\text { syst. }) \pm 0.0014(\text { ext. }) \\
a_{2} m_{+}=-0.024 \pm 0.013(\text { stat } .) \pm 0.009(\text { syst } .) \pm 0.002(\text { ext. }) .
\end{array}
$$

In addition to the statistical, systematic and external errors, these values are affected by a theoretical uncertainty which results from neglecting higher order rescattering terms. We take the difference 
between the values obtained from fit $B B$ and those obtained from the corresponding fit to the CI formulation [7] as an estimate of the theoretical uncertainty. This procedure gives a theoretical uncertainty of $\pm 0.0088( \pm 3.4 \%)$ for $\left(a_{0}-a_{2}\right) m_{+}$, and a much larger uncertainty of $\pm 0.015( \pm 62 \%)$ for $a_{2} m_{+}$.

If we use the ChPT constraint (see Eq. (3.3)), we obtain

$$
\left.\left(a_{0}-a_{2}\right) m_{+}=0.2633 \pm 0.0024(\text { stat. }) \pm 0.0014 \text { (syst. }\right) \pm 0.0019(\text { ext. }) .
$$

For this fit the theoretical uncertainty affecting the value of $a_{0}-a_{2}$ is estimated to be $\pm 2 \%( \pm 0.0053)$ from a recent study of the effect of adding three-loop diagrams to the $K^{ \pm} \rightarrow \pi^{ \pm} \pi^{0} \pi^{0}$ decay amplitude [17] in the frame of the CI formulation [7].

The obtained value of $a_{0}-a_{2}$ agrees with the results of DIRAC experiment [14], with the most recent analysis of $K_{e 4}$ decays sample, also collected by the NA48/2 collaboration [16], and with the theoretical calculations performed in the framework of Chiral Perturbation Theory [18, 19], which predict $\left(a_{0}-a_{2}\right) m_{+}=0.265 \pm 0.004$.

\section{References}

[1] J.R. Batley et al. (NA48/2), Phys. Lett. B638, 22 (2006), hep-ex/ 0606007

[2] J.R. Batley et al. (NA48/2), Phys. Lett. B634, 474 (2006), hep-ex/ 0602014

[3] J.R. Batley et al. (NA48/2), Eur. Phys. J. C52, 875 (2007), 0707.0697

[4] J.R. Batley et al. (NA48/2), Phys. Lett. B633, 173 (2006), hep-ex/ 0511056

[5] P. Budini, L. Fonda, Phys. Rev. Lett. 6, 419 (1961)

[6] N. Cabibbo, Phys. Rev. Lett. 93, 121801 (2004), hep-ph / 0405001

[7] N. Cabibbo, G. Isidori, JHEP 03, 021 (2005), hep-ph / 0502130

[8] G. Colangelo, J. Gasser, B. Kubis, A. Rusetsky, Phys. Lett. B638, 187 (2006), hep-ph / 0604084

[9] M. Bissegger, A. Fuhrer, J. Gasser, B. Kubis, A. Rusetsky, Nucl. Phys. B806, 178 (2009), 0807.0515

[10] V. Fanti et al. (NA48), Nucl. Instrum. Meth. A574, 433 (2007)

[11] G.D. Barr et al. (NA48), Nucl. Instrum. Meth. A370, 413 (1996)

[12] C. Amsler et al. (Particle Data Group), Phys. Lett. B667, 1 (2008)

[13] J.R. Batley et al. (NA48/2), Phys. Lett. B649, 349 (2007), hep-ex/ 0702045

[14] B. Adeva et al. (DIRAC), Phys. Lett. B619, 50 (2005), hep-ex/ 0504044

[15] G. Colangelo, J. Gasser, H. Leutwyler, Phys. Rev. Lett. 86, 5008 (2001), hep-ph/ 0103063

[16] B. Bloch-Devaux, PoS Confinement8, 029 (2008), http://pos.sissa.it//archive/conferences/077/029/Confinement8_029.pdf

[17] S. Gallorini, Master's thesis, Pisa University, Italy (2008), http://etd.adm.unipi.it/theses/available/etd-09012008-095804/

[18] G. Colangelo, J. Gasser, H. Leutwyler, Phys. Lett. B488, 261 (2000), hep-ph / 0007112

[19] G. Colangelo, J. Gasser, H. Leutwyler, Nucl. Phys. B603, 125 (2001), hep-ph / 0103088 